\title{
Enhanced Uptake of Luminescent Quantum Dots by Live Cells Mediated by a Membrane-Active Peptide
}

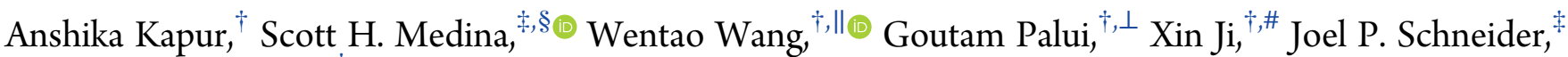 \\ and Hedi Mattoussi*, ${ }^{*} \odot$ \\ ${ }^{\dagger}$ Department of Chemistry and Biochemistry, Florida State University, 95 Chieftan Way, Tallahassee, Florida 32306, United States \\ ${ }^{\ddagger}$ Center for Cancer Research, National Cancer Institute, Building 376, Room 104, Frederick, Maryland 21702-1201, United States
}

\section{Supporting Information}

ABSTRACT: The steady progress made over the past three decades in growing a variety of inorganic nanomaterials, with discreet control over their size and photophysical properties, has been exploited to develop several imaging and sensing applications. However, full integration of these materials into biology has been hampered by the complexity of delivering them into cells. In this report, we demonstrate the effectiveness of a chemically synthesized anticancer peptide to facilitate the rapid delivery of luminescent quantum dots (QDs) into live cells. We combine fluorescence imaging microscopy, flow cytometry, and specific endocytosis inhibition experiments to probe QD-peptide conjugate uptake by different cell lines. We consistently find that a sizable fraction of the internalized conjugates does not co-localize with endosomes or the nuclei. These findings are extremely promising for the potential integration of various nanomaterials into biological systems.

\section{INTRODUCTION}

Nanoscale colloids such as those made of luminescent quantum dots (QDs) and magnetic nanocrystals, with their unique photophysical properties, are the cornerstone around which arrays of biological imaging, sensing, and tracking modalities have been developed, and they are fundamental to new diagnostics and therapeutic paradigms in precision medicine. $^{1-10}$ Among the set of developed nanoparticles (NPs) that have a great potential for advancing biological imaging and sensing, colloidal QDs are particularly appealing, due to size-tunable broad absorption spectra, narrow emission profiles, high quantum yield, large achievable Stoke's shift, and a remarkable resistance to photobleaching. These properties when combined make these materials a highly attractive alternative to organic dyes and fluorescent proteins for use in multicolor imaging and single molecule tracking. ${ }^{1,5,6,8,11-14}$ However, despite these promises, use of QDs in intracellular imaging, sensing, and drug delivery has met several limitations. Indeed, the size and nature of these nanomaterials often require that they employ active transport mechanisms to enter cells, which include nonspecific macropinocytosis and receptor-mediated uptake of these ligand-functionalized scaffolds. This can result in intracellular sequestration of the delivered nanomaterials within endosomal compartments. Alternative approaches that have been explored recently include use of chemical agents to disrupt endosomes following uptake or mechanical permeabilization of cell membranes (e.g., microinjection and electroporation). ${ }^{15-18}$ However, these strategies require the use of exogenous reagents and stimuli that can induce NP aggregation, cell damage, and release of endosomal contents; these strategies are problematic or impractical to deploy in vivo. ${ }^{8,17}$

Display of cell-penetrating peptides (CPPs) on the NP surfaces is an attractive strategy that can potentially promote their transport across the cell membrane without disrupting the lipid bilayer. Initial studies have employed several derivatives of the trans-activator of transcription (TAT) peptide from the human immunodeficiency virus-1 (HIV-1), which was coordinated to the surface of the nanoparticles. ${ }^{17,19-21}$ Various studies, including our work, have suggested that NP-CPP conjugates enter cells through a combination of endocytosis and physical translocation. . $^{9,20,22-24}$ Subsequent work combined CPP with an endosomal disruption motif in a modular peptide, JB577, to afford a partial QD-conjugate escape into the cytoplasm of treated cells. ${ }^{21,25}$ A similar approach was employed in another study where the use of arginine-rich PR9 peptide was reported to facilitate uptake followed by endosomal escape of NPs into the cell cytoplasm. ${ }^{26}$ An interesting approach that does not rely on CPPs for uptake has recently been reported by Gonzalez-Gaitan, Matile and coworkers. ${ }^{27}$ They used a biotin-modified polydisulfide coating, which was attached to commercially available streptavidinmodified QDs. Using confocal fluorescence microscopy combined with single particle tracking, they showed that incubation of these QDs with Drosophila S2 cells promoted a sizable uptake and QD delivery. QD staining was observed in all exposed cells, with an average of $\sim 70$ QDs delivered per

Received: October 23, 2018

Accepted: November 23, 2018

Published: December 12, 2018 
a

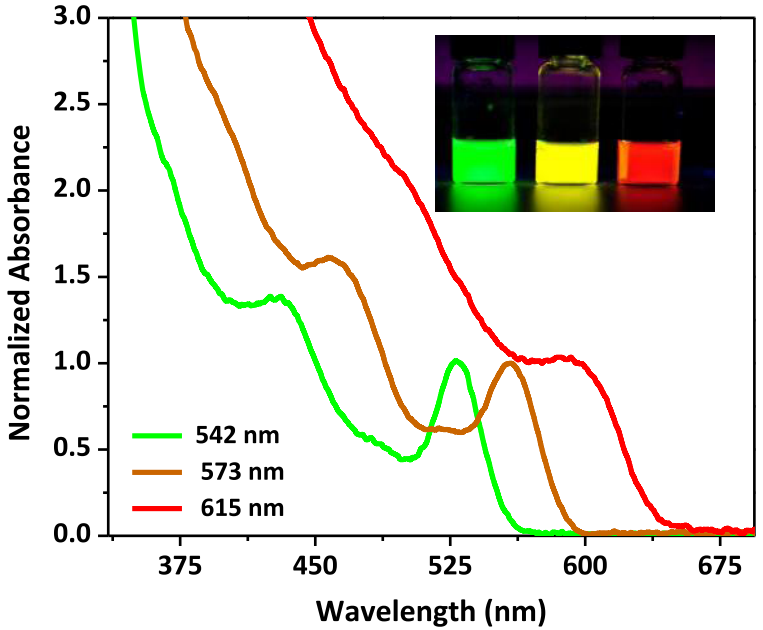

b
C
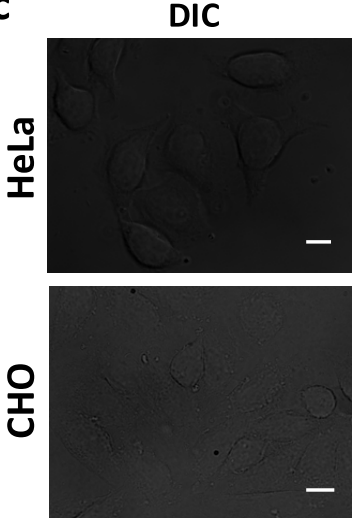

DAPI
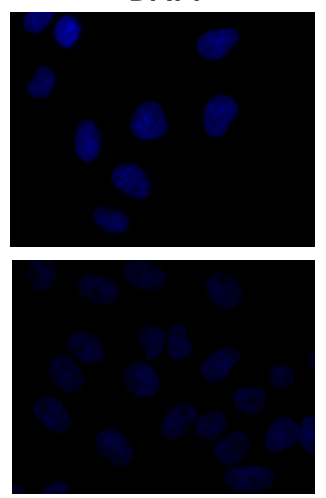

Endosome
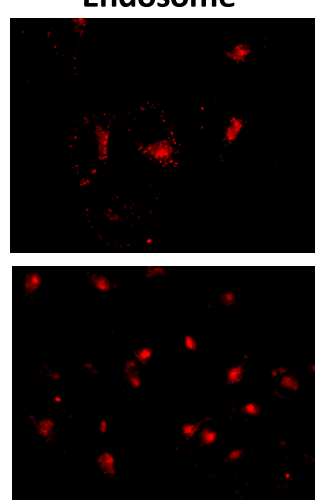

QD-SVS-1
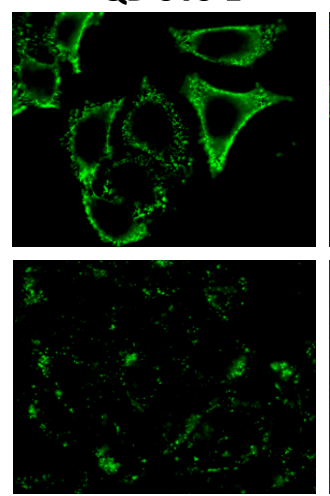

Merge
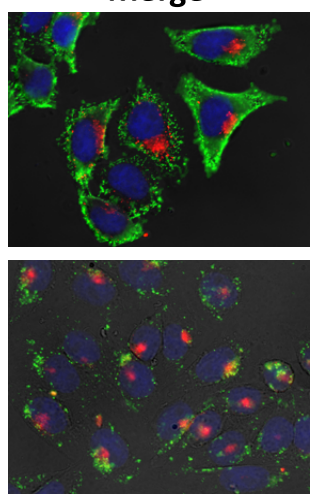

Figure 1. (a) Absorption spectra normalized with respect to the band edge peak, along with fluorescence images of green-, yellow-, and redemitting hydrophilic QDs. (b) Schematics of a QD-SVS-1 conjugate; the LA-PEG-SVS-1 structure is shown. (c) Representative fluorescence images showing conjugate internalization by HeLa and $\mathrm{CHO}$ cell lines. Cells were co-incubated for $1 \mathrm{~h}$ at $37^{\circ} \mathrm{C}$ with green 5\%-QD-SVS-1 at 50 $\mathrm{nM}$ and Texas-Red-transferrin marker, washed, then fixed, and stained with 4',6-diamidino-2-phenylindole (DAPI). Shown are the images of DAPI (blue, nuclei), endosomes (red), QDs (green) and the merged images with the differential interference contrast. Merged images show that the QD signal is distinct from that of the endosomal marker. Scale bar $\sim 10 \mu \mathrm{m}$.

probed cell. Additionally, most internalized QDs exhibited diffusive mobility and were excluded from the nucleus. Uptake for this system was attributed to the combination of two processes, one involving counterion activators and the other relying on dynamic disulfide exchange with thiols available on cell membranes. ${ }^{28}$ These investigations combined indicate that an effective intracellular delivery system may benefit from the development of new peptide sequences that can potentially interact with the cell membranes and enhance intracellular uptake to regions not limited to endosomes.

Here, we report on the ability of the de novo designed SVS1 peptide to promote a pronounced and rapid uptake of QDSVS-1 conjugates into cells. SVS-1 is a lysine-rich 18 amino acid peptide (KVKVKVKV $\left.{ }^{\mathrm{D}} \mathrm{PPTKVKVKVK}-\mathrm{NH}_{2}\right)$ that was designed to interact with the negatively charged membranes of cancer cells. It is different from other often arginine-rich CPPs, such as TAT. Initial results indicated that a large fraction of SVS-1 incubated with cancer cells crossed the membrane into the cytoplasm, leading to their lytic destruction. ${ }^{29}$ Conversely, cells incubated with the peptide at concentrations smaller than $\mathrm{IC}_{50}$ value (half-maximal inhibitory concentration) were not affected. It was proposed that SVS-1 rather enters cells, with the majority of peptides translocating directly across the membrane into the cytoplasm. ${ }^{30}$ Since the mechanism and efficiency of peptide uptake tend to depend on the cargo and cell type, we reasoned that coupling several copies of SVS-1 to luminescent QDs could mediate their rapid and efficient internalization into live cells. We test the effects of varying the QD-SVS-1 valence, nanocrystal size, concentration, incubation time, and nature of the cells used on the degree of uptake for four different cell lines. We have also attempted to identify the distribution of conjugates following uptake and performed preliminary endocytosis inhibition tests to gain some insights into the mechanistics of the QD-conjugate intracellular uptake.

\section{RESULTS AND DISCUSSION}

We prepared three different sets of $\mathrm{CdSe}-\mathrm{ZnS}$ core-shell QDs with fluorescence peaks centered at 542, 572, and 618 $\mathrm{nm}$, following reported protocols. ${ }^{31,32}$ The absorption profiles along with a florescence image of the QD dispersions are shown in Figure 1. Phase transfer of the hydrophobic QDs to aqueous buffers was carried out via a photochemical ligation strategy in the presence of a mixture of methoxy-terminated (inert) and amine-terminated (reactive) poly(ethylene glycol) (PEG) ligands appended with lipoic acid anchors, LA-PEG$\mathrm{OCH}_{3}$ and LA-PEG- $\mathrm{NH}_{2}$, respectively. ${ }^{33,34}$ This affords control over the number of reactive amine groups on the QD surface, allowing one to tune the number of SVS-1 peptides per 
a
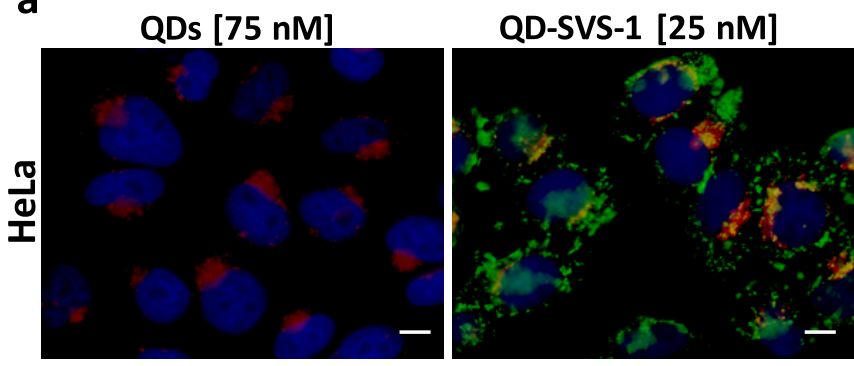

b
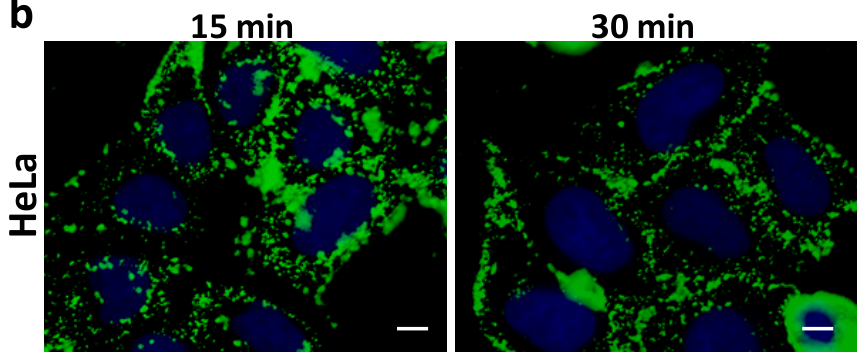



QD-SVS-1 [75 nM]
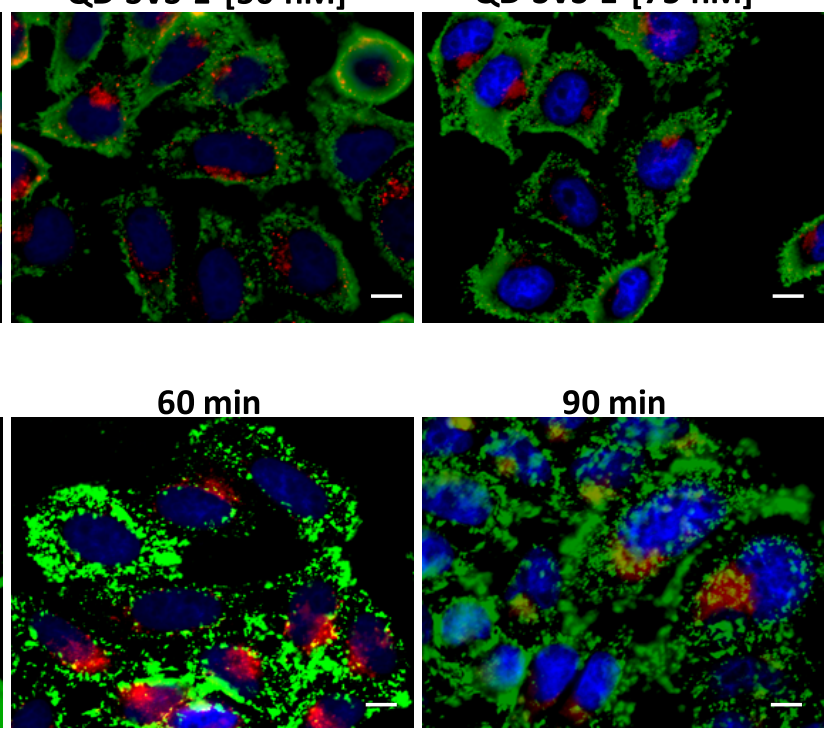

c



d

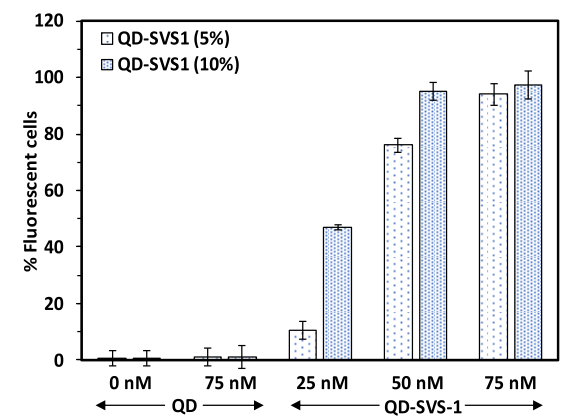

e



Figure 2. (a) Representative fluorescence images showing the concentration-dependent uptake of green 5\%-QD-SVS-1 conjugates. The cells were co-incubated with various concentrations of QD-SVS-1 and Texas-Red-transferrin for $1 \mathrm{~h}$ at $37^{\circ} \mathrm{C}$. Cells incubated with QDs at $75 \mathrm{nM}$ show no uptake. (b) Fluorescence images of HeLa cells incubated with 5\%-QD-SVS-1 (50 nM) for 15, 30, 60, and 90 min at $37^{\circ} \mathrm{C}$; co-labeling of the endosomes with Texas-Red-transferrin (red, $60 \mathrm{~min}$ incubation) was limited to cultures incubated with the conjugates for 60 and 90 min. Flow cytometry analysis of conjugate internalization for: (c) green 5\%-QD-SVS-1 by CHO cells (light gray bars) and HeLa cells (dark gray bars); (d) green 5\%-QD-SVS-1 (light gray bars) and green 10\% QD-SVS-1 (gray bars) by HeLa cells; (e) green-(light gray bars), yellow-(gray bars) and red-(dark gray bars) emitting 5\%-QD-SVS-1 conjugates by HeLa cells. All of the flow cytometry measurements were carried out using incubation for $1 \mathrm{~h}$ at $37^{\circ} \mathrm{C}$. Scale bar $\sim 10 \mu \mathrm{m}$.

conjugate. QDs with two fractions of LA-PEG- $\mathrm{NH}_{2}, 5 \%$ (5\%-QDs) and 10\% (10\%-QDs), were prepared. The available amine groups on the QDs were then modified with $\mathrm{N}$ hydroxysuccinamide-3-(3-methyl-2,5-mioxo-2,5-dihydro- $1 \mathrm{H}$ pyrrol-1-yl)propionic acid (NHS ester maleimide) and then reacted with the N-terminal cysteine-modified SVS-1, forming a stable thioether linkage (see Figure $1 \mathrm{~b}$ ). This is expected to yield a controlled number of SVS-1 per green-emitting QD, ranging from $\sim 8-10$ for the $5 \%$-QDs to $\sim 16-20$ for the $10 \%$ QDs. $^{33}$

We first probed the ability of the peptide to promote the delivery of QDs into different types of cells. Green-emitting QD-SVS-1 conjugates (starting with 5\%-QDs) were mixed with Texas-Red-labeled transferrin protein (serving as an endosome marker) and then co-incubated with four different mammalian cell lines, HeLa, A549, human umbilical vein endothelial (HUVEC), and Chinese hamster ovary (CHO) cells, for $1 \mathrm{~h}$ at $37^{\circ} \mathrm{C}$. The epifluorescence images in Figure 1c show extensive green signal from the QDs distributed across the cell volume, in particular for the HeLa cells. Additional images collected from A549 and HUVEC cells are provided in the Supporting Information, Figure S1. Furthermore, the internalized QDs were homogeneously distributed throughout the cell volume. Essentially, little to no co-localization with the endosome marker or nuclear stain was observed, as illustrated in the merged images shown in Figure 1c, right panels. However, no QD uptake was observed in control cells incubated with unconjugated QDs (5\%-QDs, no peptide) under the same conditions (see Supporting Information, Figure S2). These results suggest that the SVS-1 peptide can promote the internalization of QDs into mammalian cell lines, where the conjugates then distribute across the cell volume.

Next, we tested the effects of varying the concentration and incubation time on the internalization by HeLa and $\mathrm{CHO}$ cells using fluorescence spectroscopy imaging, supplemented by quantification of the uptake using flow cytometry measurements (additional details on the flow cytometry data and analysis are provided in the Supporting Information, Figure S3). As illustrated in Figure 2, increased QD internalization that is commensurate with the conjugate concentration used 

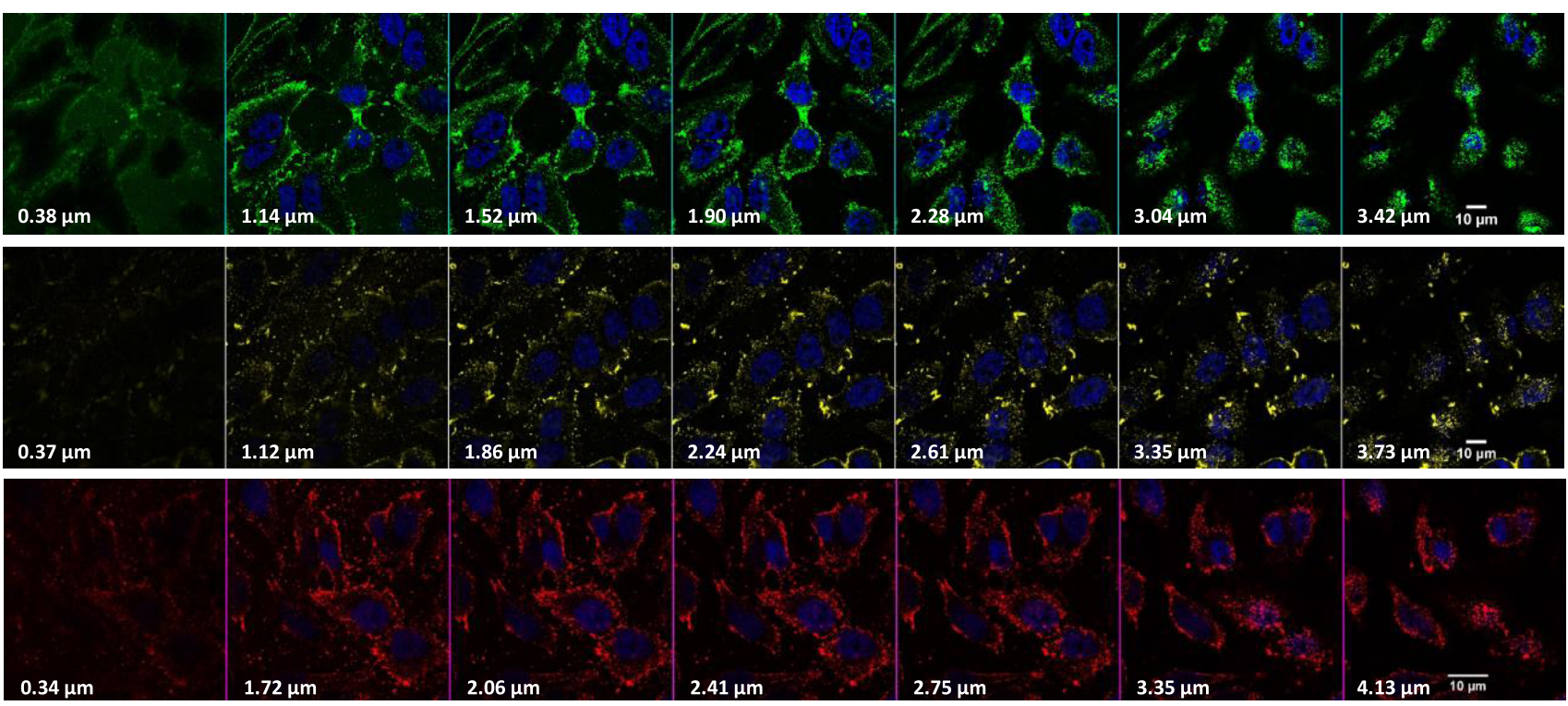

Figure 3. Representative confocal microscopy images collected from HeLa cells incubated with 5\%-QD-SVS-1 conjugates. The row of images corresponds to cells incubated with green-(top), yellow-(middle), and red-(bottom) emitting QD-conjugates at $50 \mathrm{nM}$ for $1 \mathrm{~h}$ at $37^{\circ} \mathrm{C}$. Images are acquired at the indicated position along the $z$-axis. Scale bar $\sim 10 \mu \mathrm{m}$.

(from 25 to $75 \mathrm{nM}$ ) was measured for HeLa and CHO cells. The fluorescence images in Figure $2 \mathrm{a}$ show concentrationdependent increase in the QD staining of the cells, whereas the data shown in Figure $2 \mathrm{~b}$ indicate that significant levels of conjugate internalization by the cells occurred within 15-30 min of incubation, which confirms that enhanced uptake is facilitated by the peptides. Furthermore, we measured a more pronounced uptake of QD-SVS-1 by HeLa cells compared to noncancerous $\mathrm{CHO}$ cells for all concentrations tested (Figure 2c), a finding that is consistent with the reported higher selectivity of SVS-1 peptide toward cancer cells compared to normal cell lines. ${ }^{36}$

We investigated the internalization with respect to changes in the QD-SVS-1 conjugate valence by varying the number of peptides coupled to a QD. Indeed, we found that starting with $10 \%$ QDs (expected to double the valence from $\sim 10$ to $\sim 20$ peptides per QD) resulted in enhanced internalization by HeLa cells compared to 5\% QDs, as monitored by flow cytometry measurements (Figure $2 \mathrm{~d}$ ). Taken together, these data clearly prove that the observed internalization is mediated by the display of SVS-1 peptides on the QDs. The data also demonstrate that our intracellular QD delivery scheme is effective, efficient, and rapid while maintaining the selective properties of the peptide.

The effects of varying the QD surface area on the degree of internalization were investigated by incubating the cells with conjugates prepared using three nanocrystal sizes (radius $\sim 3.2$, $\sim 3.4$, and $\sim 3.8 \mathrm{~nm}$ for green, yellow, and red QDs, respectively). ${ }^{37,38}$ In particular, the three sets of $5 \%-\mathrm{NH}_{2}-$ QDs were conjugated to SVS-1. By virtue of the increased area with size, this would yield conjugates with increasing numbers of SVS-1 per nanocrystal from green to red. We anticipate, based on simple geometrical consideration and using the density of $\mathrm{LA}-\mathrm{PEG}-\mathrm{NH}_{2}$ ligands per $\mathrm{QD}$, that the numbers of coupled peptides per nanocrystal are $\sim 10,12$, and 15 for green, yellow, and red QDs, respectively, starting with 5\% amine-QDs. ${ }^{35}$ The experimental results collected from flow cytometry measurements (shown in Figure 2e) confirm that SVS-1 effectively mediates cellular delivery of QDs with higher uptake for larger NCs (i.e., larger surface area, red > yellow > green), as anticipated. Moreover, the difference in uptake is more pronounced at lower concentrations, where subsaturation in uptake better reflects the influence of increased conjugate valence. At higher concentrations, however, the degree of cellular uptake seems to reach saturation, reducing the effects of conjugate valence on the degree of uptake. Additional fluorescence images of HeLa cells incubated with red QD-conjugates are shown in Figure S4. To confirm that the observed QD staining was indeed emanating from QDs inside the cytoplasm and not originating from membranebound conjugates, we collected a set of confocal microscopy images from the cell cultures. Representative confocal images of HeLa cells incubated with green-, yellow-, and red-emitting $5 \%$-QD-SVS-1 conjugates are shown in Figure 3. The images collected from the three sets of QDs strongly suggest that the QD staining is distributed throughout the cell volumes and outside the nuclei.

To confirm that the intracellular delivery data collected using postuptake fluorescence imaging is not an artifact caused by cell fixation, we carried out live cell imaging where conjugate internalization could be tracked in situ. For this, HeLa cells were first incubated with red-emitting QD-SVS-1 conjugates (starting with $5 \%-\mathrm{NH}_{2}-\mathrm{QDs}$ ) at a concentration of $50 \mathrm{nM}$, then fluorescence image collection was initiated immediately after exposure to the conjugates and continued for a period of $30 \mathrm{~min}$ (one frame every $\sim 2.5 \mathrm{~min}$ ). The compiled video shows that red $\mathrm{QD}$ staining could be detected immediately after exposure to the conjugates and continued to expand throughout the collection period (see Movie S1). This provides further evidence for the ability of the surfacedisplayed peptide to enable the rapid delivery of the QD cargos inside cells.

The above results combined strongly suggest that the lysinerich, foldable SVS-1 peptide is very effective in delivering conjugated QDs into live cells. This has further motivated the idea of testing whether or not translocation of these conjugates inside the cells is solely dependent on endocytosis. For this, we carried out three uptake experiments in the presence of known 
a
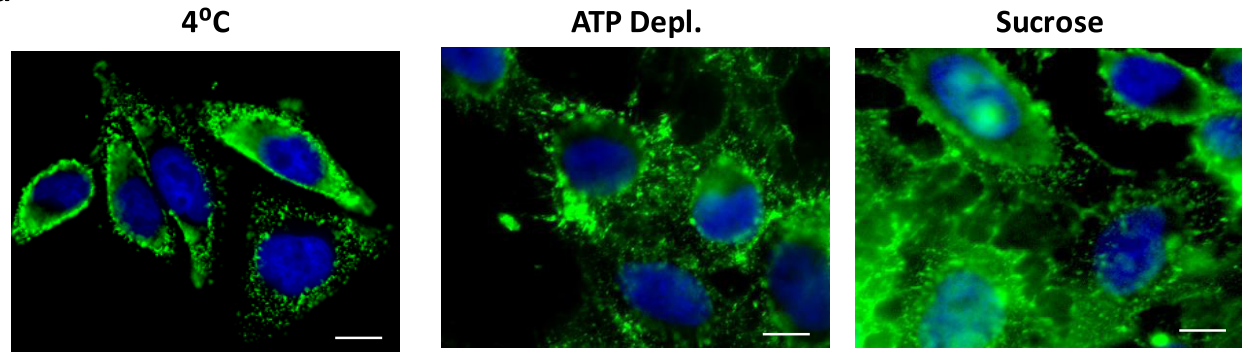

b

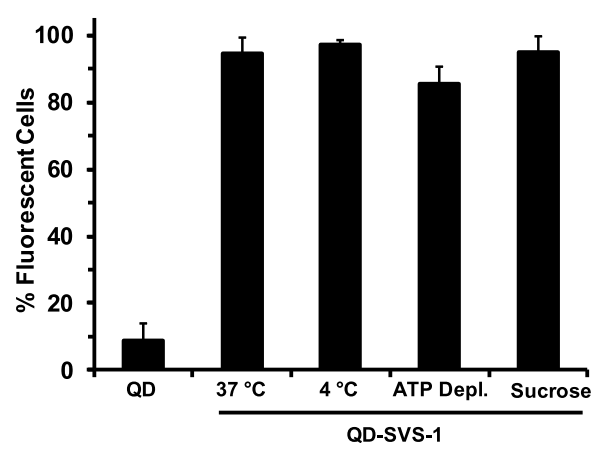

Figure 4. (a) Representative fluorescence images of HeLa cells incubated with QD-SVS-1 (50 nM). (b) Side-by-side comparison of cell staining (from flow cytometry measurements) following incubation with 5\%-QD-SVS-1 (50 nM) for 40 min under endocytosis inhibition conditions, together with control conditions at $37{ }^{\circ} \mathrm{C}$ and incubation with unconjugated QDs. Scale bar $\sim 10 \mu \mathrm{m}$.

physical and pharmacological inhibitors of endocytosis. In the first, the temperature of the cell culture was adjusted to $4{ }^{\circ} \mathrm{C}$ for $40 \mathrm{~min}$, and then incubation with the $\mathrm{QD}$-peptide conjugates was carried out for an additional $40 \mathrm{~min}$. Lowering the temperature to $4{ }^{\circ} \mathrm{C}$ is known to deplete the cell's energy, thus eliminating clathrin-mediated endocytosis as an uptake process. ${ }^{2,20}$ In the second, we tested the effects of chemical inhibition. The cell culture was initially pretreated with sodium azide $\left(\mathrm{NaN}_{3}, 10 \mathrm{mM}\right)$ and $50 \mathrm{mM}$ 2-deoxy-D-glucose for 30 min before incubation with the conjugates. In the third test, the culture was treated with a hypertonic sucrose solution $(0.4$ M) for $1 \mathrm{~h}$, then incubated with the QD-SVS-1 conjugates for $40 \mathrm{~min}$. The three sets of cell cultures were then fixed and imaged using epifluorescence microscopy. Incubation with 2deoxy-D-glucose and $\mathrm{NaN}_{3}$, respectively, inhibits glycolysis and mitochondrial oxidative phosphorylation, leading to impairment of ATP production (thus altering the active process of endocytosis). Conversely, exposure to a hypertonic solution of sucrose specifically inhibits clathrin-mediated endocytosis. ${ }^{39-41}$ Figure $4 \mathrm{a}$ shows that significant levels of QD staining are measured inside the cells exposed to the conjugates under all of the above conditions. Additional flow cytometry measurements of cells incubated with QD-SVS-1 (5\%-QDs) allowed us to compare the cellular uptake in the presence and absence of endocytosis inhibitory conditions. Negligible differences were detected in the percentage of fluorescent cells between treated and nontreated cultures, as shown in Figure $4 \mathrm{~b}$.

We would like to stress that incubation with the conjugates did not induce any measurable toxicity to the various cells, as confirmed using 3-(4,5-dimethylthiazol-2-yl)-2,5-diphenyltetrazolium bromide (MTT) viability tests. Figure 5 shows that indeed the viability of the cells was essentially unchanged following incubation with the QD-SVS-1 conjugates (or QDs

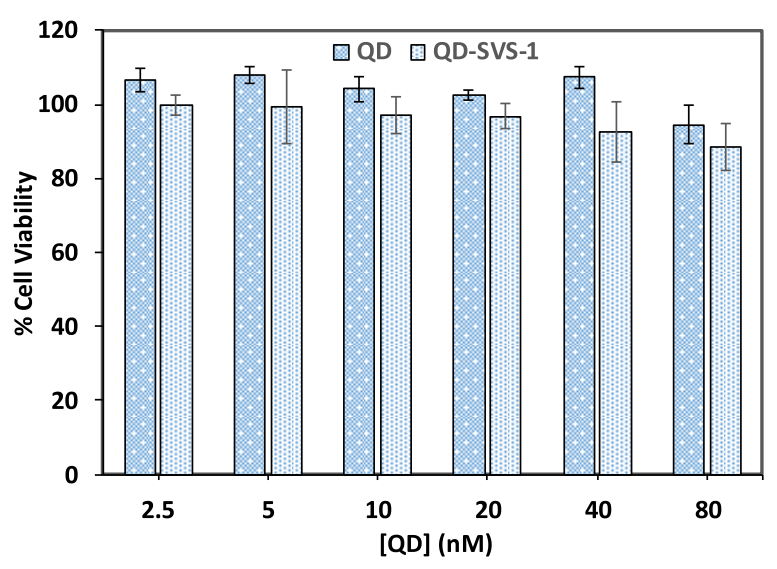

Figure 5. MTT viability test applied to HeLa cells incubated with QDs and QD-SVS-1 conjugates. Green-emitting unconjugated QDs or 5\%-QD-SVS-1 conjugates were used.

alone) over the range of concentrations used in the uptake experiments.

Finally, we should note that the effectiveness of the SVS-1 peptide for promoting the rapid uptake of other types of nanomaterials, namely, Au nanoparticles (AuNPs) and large Au nanorods (AuNRs), has also recently been reported by our group. ${ }^{42}$ The metal nanostructures were coated with a multicoordinating polymer ligand; such coating promotes long-term steric stability of the nanocrystals, while providing a large number of reactive amine groups per NP/NR. This is particularly important for nanorods, which have rather larger overall size and surface areas, and the polymer provides better interfacing with the surrounding medium than monomeric ligands. Additionally, given the fact that AuNPs and AuNRs are inherently not fluorescent and tend to quench the emission of 
proximal dyes and other fluorescing materials, the polymer coating with its multireactive sites was coupled to a large number of Texas-Red dyes along with several copies of SVS1. ${ }^{42}$ This provided the means to visualize the uptaken AuNPand AuNR-conjugates. We found that incubation of the $\mathrm{Au}$ nanocrystal-SVS-1 conjugates produced rapid and pronounced conjugate delivery, yielding extended red staining across the cell volumes. The homogenous and extensive staining was excluded from the cell nuclei. Furthermore, endocytosis inhibition experiments, similar to those performed here for the QDs, were carried out, namely, using incubation at $4{ }^{\circ} \mathrm{C}$, pretreatment in the presence of 2-deoxy-D-glucose and $\mathrm{NaN}_{3}$ mixture, or with sucrose. A pronounced uptake of AuNP- and AuNR-conjugates was measured under those conditions, a result consistent with the data shown in Figure 4 above.

\section{CONCLUSIONS}

In summary, our results combining fluorescence imaging, flow cytometry, and endocytosis inhibition measurements show that the membrane-active peptide (SVS-1) can promote rapid and pronounced delivery of QD-conjugates into live cells. The intracellular uptake can further be controlled by varying the conjugate concentration, density of surface-displayed peptides, and nature of the cell lines, with cancerous cell cultures exhibiting higher degree of internalization than noncancerous ones. Importantly, the internalized QDs do not show colocalization with apo-transferrin endosome marker, and uptake of these QD nanomaterials persists when incubation was carried out in the presence of certain endocytosis inhibitors. Nonetheless, because apo-transferrin marker is known to primarily label the recycling endosomes of cells, interaction with other types of endocytic vesicles cannot be discarded. These findings, though preliminary, are promising as they open up new possibilities for addressing the limitations currently encountered in delivering various nanomaterials into live cells, without relying on endocytosis. Our scheme offers a more facile and potentially direct approach for the rapid intracellular delivery of nanoconjugates into various cell lines. Our studies suggest that one of the possible mechanisms of cell entry could involve direct translocation across the membrane. ${ }^{43}$ Nonetheless, further studies are needed to fully understand the mechanism governing the cellular uptake.

We would also like to mention that the QD staining, though distributed across the cells, also seems to be punctate. This could be promoted by postinternalization interactions of the peptide with the membrane-enclosed organelles. To gain further understanding of the exact mechanism, test its effectiveness for promoting the uptake of other nanomaterials, we are performing additional studies to co-label the subcellular organelles and identify any post internalization interactions. Use of other nanoparticle-SVS-1 conjugates (e.g., AuNPs and AuNRs), ${ }^{42}$ combined with high-resolution imaging tools, like transmission electron microscopy and scanning electron microscopy, will expand the applicability of this approach and further provide additional information on the localization and uptake mechanism. Finally, exploring the use of mixed surface conjugates combining SVS-1 with other biomolecules (such as mRNA and DNA) will allow us to expand on existing platforms for subcellular delivery.

\section{EXPERIMENTAL SECTION}

QD Growth and Ligand Exchange. The $\mathrm{CdSe}-\mathrm{ZnS}$ core-shell QDs used in this study were prepared in two steps. ${ }^{31}$ The CdSe core was grown first by reduction of cadmium and selenium organometallic precursors at high temperature $\left(300-350{ }^{\circ} \mathrm{C}\right)$ and in coordinating solvent mixtures made of alkylphosphines, alkyphosphine-carboxyl, and alkylamines. This was followed by overcoating the CdSe core with a $\mathrm{ZnS}$ shell, also using organometallic (zinc and sulfur) precursors but at lower temperature. ${ }^{31}$ The nanocrystal core size was controlled by adjusting the precursor concentrations and temperature; overall, the same thickness of the overcoating $\mathrm{ZnS}$ layer was maintained for all samples.

The hydrophobic QDs were phase-transferred to buffer media by photoligation with a mixture of LA-PEG-OCH and $\mathrm{LA}-\mathrm{PEG}-\mathrm{NH}_{2}$ ligands. We describe the preparation of green-emitting QDs photoligated with 5\% LA-PEG- $\mathrm{NH}_{2}$ (5\%-QDs). ${ }^{33}$ Hydrophobic QDs $(150-200 \mu \mathrm{L}$, from a stock QD dispersion, 15-20 $\mu \mathrm{M}$ ) were precipitated using ethanol. The dispersion was centrifuged for $10 \mathrm{~min}$ at $3000 \mathrm{rpm}$. The supernatant was discarded, and the pellet was resuspended in $750 \mu \mathrm{L}$ of hexane. Separately, $4.2 \mathrm{mg}$ of LA-PEG- $\mathrm{NH}_{2}, 95$ mg of $\mathrm{LA}-\mathrm{PEG}-\mathrm{OCH}_{3}$, and $\sim 1 \mathrm{mg}$ of tetramethylammonium hydroxide were dissolved in $500 \mu \mathrm{L}$ of methanol and added to the QD dispersion. This QD-plus-ligand mixture (two phase) was placed in a UV photoreactor (Model LZC-4 V, Luzchem Research Inc., Ottawa, Canada) and irradiated for $40 \mathrm{~min}$ (at $350 \mathrm{~nm}, 4.5 \mathrm{~mW} / \mathrm{cm}^{2}$ ) while stirring. The methanol layer containing the QDs was separated and slightly dried under vacuum. A mixture of methanol, chloroform, and hexane was added to the QDs at a volume ratio of $1: 1: 10$ and then centrifuged for $10 \mathrm{~min}$ at $3000 \mathrm{rpm}$. The precipitated QDs were gently dried under vacuum and redispersed in $1 \mathrm{~mL}$ of $\mathrm{H}_{2} \mathrm{O}$. The dispersion in water was passed through a $0.45 \mu \mathrm{m}$ syringe filter (Millipore, Billerica, MA) and further purified by applying three rounds of concentration/dilution using a centrifugal membrane filtration device (MW cutoff $50 \mathrm{kDa}$, Millipore, Billerica, MA). The same protocol with a few adjustments in the amounts of ligands used was applied to prepare green-emitting QDs with 10\% LA-PEG- $\mathrm{NH}_{2}$ (10\%QDs) as well as yellow- and red-emitting QDs with 5\% LAPEG $-\mathrm{NH}_{2}{ }^{34}$ The final concentrations of the QD dispersions were determined by combining the absorbance values and the molar extinction coefficients at $350 \mathrm{~nm}, \varepsilon_{350}$. Values of $8.1 \times$ $10^{5}, 10.7 \times 10^{5}$, and $1.96 \times 10^{6} \mathrm{M}^{-1} / \mathrm{cm}^{-1}$ were used for the extinction coefficients of the green-, yellow-, and red-emitting QDs. $^{38,44,45}$

Preparation of QD-SVS-1 Peptide Conjugates. The surface-tethered amines on the QDs were coupled to the $\mathrm{N}$ terminal cysteine residue on the peptide via NHS ester maleimide coupling chemistry. ${ }^{46}$ Here, we limit our description to the conjugation of green-emitting 5\%-QDs to SVS-1 peptide. A similar protocol was applied to prepare yellowand red-emitting QD-SVS-1 conjugates. Briefly, $0.24 \mathrm{mg}$ of SVS-1 peptide was dissolved in $150 \mu \mathrm{L}$ of tris-buffered saline (TBS, pH 7.3) and mixed with $7.5 \mu \mathrm{L}$ of $6.98 \mathrm{mM}$ solution of tris(2-carboxyethyl)phosphine (TCEP) (the molar ratio with respect to peptide was $\sim 0.5$ ), and then the mixture was left stirring at room temperature for $15 \mathrm{~min}$. In a separate glass vial, $100 \mu \mathrm{L}$ of $5 \%$-QDs $(10.7 \mu \mathrm{M})$ was dispersed in $200 \mu \mathrm{L}$ of phosphate buffer ( $\mathrm{pH} 7.5,20 \mathrm{mM}$ ), and then $25 \mu \mathrm{L}$ of $40 \mathrm{mM}$ NHS ester maleimide solution in dimethyl sulfoxide (DMSO) 
was added; this corresponds to $\sim 1000$ times molar excess maleimide with respect to QDs. The reaction mixture was stirred for $\sim 30 \mathrm{~min}$, then one round of dilution/concentration with TBS buffer ( $\mathrm{pH}$ 7.3) using a membrane filtration device (MW cutoff $=50 \mathrm{kDa})$ was applied to remove excess NHS ester maleimide. The purified maleimide-modified QD dispersion was added to the peptide-TCEP mixture followed by addition of TBS ( $\mathrm{pH} 7.3$ ) to adjust the final volume to 300 $\mu \mathrm{L}$. The mixture was incubated for another $\sim 3 \mathrm{~h}$ while stirring. The QD-SVS-1 conjugates were purified from unbound peptide via the size-exclusion chromatography using a PD-10 column (GE Healthcare, Piscataway, NJ), then stored at $4{ }^{\circ} \mathrm{C}$ until further use. We anticipate that this protocol would yield an average of $\sim 10$ peptides per QD-conjugate.

Cell Culture and Fluorescence Imaging Experiments. Four cell lines were used in this study. Human cervical carcinoma (HeLa) cells were acquired from the cell culture facility at Florida State University; Chinese hamster ovary ( $\mathrm{CHO}$ ) cells were kindly provided by Strouse Laboratory at FSU; human umbilical vein endothelial (HUVEC) and adenocarcinoma human alveolar basal epithelial (A549) cells were provided by the NCI-60 repository (Frederick, MD). The cell cultures were grown at $37{ }^{\circ} \mathrm{C}$ under humidified $5 \% \mathrm{CO}_{2}$ atmosphere in complete growth medium (Dulbecco's modification of Eagle medium, DMEM). The growth medium was supplemented with $10 \%(\mathrm{v} / \mathrm{v})$ fetal bovine serum, L-glutamine, sodium pyruvate, $1 \%(\mathrm{v} / \mathrm{v})$ antibiotic-antimycotic $100 \times$, and $1 \%(\mathrm{v} / \mathrm{v})$ nonessential amino acid solution $100 \times$. For incubation experiments, $7 \times 10^{4}$ cells were seeded onto microcover glasses in a 24-well microplate (CELLSTAR, VWR); cell attachment was achieved by further incubating the culture overnight. For the uptake studies, the cultures were mixed with nanocrystal-SVS-1 conjugates, or dispersions of the nanocrystals alone, along with Texas-Red-labeled transferrin (when necessary). The various reagents were prepared in serum-free DMEM media at the desired concentrations. After incubation, the cells were washed three times with TBS buffer, fixed with $3.7 \%$ paraformaldehyde, and then stained with DAPI to allow visualization of the nuclei.

Experiments testing inhibition of endocytosis were carried out using cells that had been cultured overnight. Three sets of inhibition experiments were carried out. (1) The cell cultures were first preincubated at $4{ }^{\circ} \mathrm{C}$ for $40 \mathrm{~min}$, prior to mixing with the conjugates. ${ }^{47}$ (2) The ATP depletion experiments were carried out by first incubating the cells in glucose and serumfree media containing $10 \mathrm{mM}$ sodium azide and $50 \mathrm{mM} \mathrm{2-}$ deoxy-D-glucose for $30 \mathrm{~min}$, prior to mixing with the QDconjugates. $^{48}(3)$ The cells were preincubated for $1 \mathrm{~h}$ in serumfree media containing $0.45 \mathrm{M}$ sucrose; these conditions are expected to selectively prevent the process of clathrin-mediated endocytosis. ${ }^{49}$ In all three cases, the cell cultures were washed with serum-free media followed by incubation with the QDpeptide conjugates at the required concentration for $40 \mathrm{~min}$. The cultures were then washed, fixed, and mixed with DAPI for nuclei staining, as described above.

The fluorescence images of the various labeled and fixed cell cultures were acquired using an Inverted Nikon Eclipse $\mathrm{Ti}$ microscope equipped with a color CoolSNAP HQ2 CCD camera. Excitation of the samples was carried out using a Xe lamp, whereas the fluorescence images were collected using a $60 \times$ objective combined with a set of filter cubes acquired from Chroma Technology (Rockingham, VT). These include a DAPI cube (with $340-380 \mathrm{~nm}$ excitation and $435-485 \mathrm{~nm}$ emission) to visualize the nuclei, a green fluorescent protein/ enhanced green fluorescent protein cube (with 450-490 nm excitation and 500-550 $\mathrm{nm}$ emission) to detect the green signal from the QDs; finally, visualization of either the redemitting QDs or the Texas-Red-transferrin staining was achieved using a Texas-Red HYQ cube (with 532-587 nm excitation and 608-683 $\mathrm{nm}$ emission).

The cell cultures used for the live cell imaging studies were prepared as follows. HeLa cells $\left(2 \times 10^{4}\right)$ per well were seeded in eight-well chambered glass slides and incubated overnight to allow adherence under normal culture conditions. The cells were then washed and incubated with $2 \mu \mathrm{g} / \mathrm{mL}$ Hoechst 33342 dye for $20 \mathrm{~min}$, then mounted onto an EVOS FL Auto fluorescent microscope equipped with an environmental chamber to maintain $37{ }^{\circ} \mathrm{C}$ and $5 \% \mathrm{CO}_{2}$ during experiments (ThermoFisher Scientific, Waltham, MA). A concentrated solution of QD-SVS-1 conjugates was added to the cells to reach a final concentration of $50 \mathrm{nM}$, and the cells were immediately imaged using a $20 \times$ objective. Illumination of the culture was provided by a set of manufacturer LED light cubes for DAPI (357/44 nm excitation, 447/60 nm emission) and Texas-Red (585/29 nm excitation, 628/32 nm emission). Images were collected every $2.5 \mathrm{~min}$ over a total period of 30 min to monitor intracellular internalization of the conjugates.

Flow Cytometry Measurements. The cell cultures used for performing the flow cytometry experiments were prepared by seeding $1.6 \times 10^{5} \mathrm{HeLa}$ cells/well in a 24 -well plate and allowing the cells to adhere overnight under normal culture conditions. Flow cytometry experiments applied to the QDs tested the effects of concentration, conjugate valence, QD size, and selectivity. The cells were washed with TBS, then $0.5 \mathrm{~mL}$ serum-free media containing unconjugated QDs or QD-SVS1 conjugates (diluted to required concentrations) was added, followed by incubation for $1 \mathrm{~h}$ at $37{ }^{\circ} \mathrm{C}$. After treatment, the cells were washed with TBS and incubated with $150 \mu \mathrm{L}$ of $0.25 \%$ trypsin-ethylenediaminetetraacetic acid solution for 15 $\mathrm{min}$. This procedure allows to both collect the cells for analysis and digest any QD-conjugates that were not internalized but nonspecifically adsorbed onto the cell membranes. ${ }^{49}$ The cells were pelleted by centrifugation at $2000 \mathrm{rpm}$ for $5 \mathrm{~min}$ and then resuspended in $1 \mathrm{~mL}$ of fresh TBS. The samples were analyzed using a BD FACSCanto RUO Special Order System flow cytometer using 488 and $561 \mathrm{~nm}$ laser lines for excitation of the green- and yellow/red-emitting QDs, respectively. Gating was based on normalized fluorescence of untreated cells to evaluate the fraction of cells that have internalized the QDSVS-1 conjugates. Uptake studies were performed in three independent experiments using three replicates for each experimental condition.

To determine the role of endocytosis on QD-SVS-1 conjugate internalization, incubation of the cells was tested under endocytosis inhibition conditions. Three conditions were explored. Cell cultures were incubated at $4{ }^{\circ} \mathrm{C}$ or in the presence of two chemical inhibitors (sodium azide or sucrose), as described above. The cells were washed with serum-free media followed by incubation with media containing greenemitting QD-SVS-1 conjugates at $50 \mathrm{nM}$ for $40 \mathrm{~min}$. The cultures were then prepared for flow cytometry analysis, as described above. Measurements were carried out using the Beckman Coulter FACSCalibur flow cytometer equipped with $488 \mathrm{~nm}$ laser source for fluorescence excitation.

Cell Viability Assay. The viability test was limited to HeLa cells. The cell cultures were incubated with either uncon- 
jugated green QDs or green 5\%-QD-SVS-1 conjugates, and their viability was assessed using MTT assay. Briefly, HeLa cells were seeded onto 96 -well microplates $\left(3 \times 10^{4}\right.$ cells $/ 200$ $\mu \mathrm{L} /$ well) in triplicates and allowed to adhere overnight. The media were replaced with $150 \mu \mathrm{L}$ of media containing dispersions of either QDs alone or QD-SVS-1 conjugates at concentrations ranging from 2.5 to $80 \mathrm{nM}$. A negative control was also prepared by incubating cells with media containing $20 \%$ DMSO. After $24 \mathrm{~h}$ incubation, the cells were washed with PBS three times and then $100 \mu \mathrm{L}$ of freshly prepared MTT solution in serum-free media $(0.5 \mathrm{mg} / \mathrm{mL})$ was added to each well. The cultures were further incubated at $37{ }^{\circ} \mathrm{C}$ for $3 \mathrm{~h}$. The MTT solution in each well was replaced with $150 \mu \mathrm{L}$ of DMSO, and incubated for 30 min until the MTT-formazan product was completely solubilized. The absorbance was measured at $560 \mathrm{~nm}$ using a microplate reader (Infinite M1000 PRO from TECAN). The viability was calculated and expressed as a percentage with respect to the absorbance of the control culture (i.e., cells not treated with QDs or QDSVS-1).

\section{ASSOCIATED CONTENT}

\section{S Supporting Information}

The Supporting Information is available free of charge on the ACS Publications website at DOI: 10.1021/acsomega.8b02918.

Additional details about the materials used; peptide synthesis; ligand synthesis; fluorescence imaging of control samples and flow cytometry data (PDF)

Red quantum dot staining (AVI)

\section{AUTHOR INFORMATION}

\section{Corresponding Author}

*E-mail: mattoussi@chem.fsu.edu.

\section{ORCID}

Scott H. Medina: 0000-0001-5441-2164

Wentao Wang: 0000-0003-2273-4171

Hedi Mattoussi: 0000-0002-6511-9323

\section{Present Addresses}

\#Ocean Nanotech, LLC, 7964 Arjons Drive, San Diego, California 92126, United States (X.J.).

${ }^{\perp}$ The Food and Drug Administration, National Center for Toxicological Research, 3900 NCTR Road, Jefferson, Arkansas 72079, United States (G.P.).

"DNA Electronics, Inc., 1891 Rutherford Road Suite 100, Carlsbad, California 92008, United States (W.W.).

${ }^{\S}$ Department of Biomedical Engineering, Pennsylvania State University, University Park, State College, Pennsylvania 16802, United States (S.H.M.).

\section{Notes}

The authors declare no competing financial interest.

\section{ACKNOWLEDGMENTS}

This work was supported by FSU, the National Science Foundation (NSF-CHE \#1508501 and \#1058957), the National Institutes of Health (NIH \#R01 DC013080), Asahi-Kasei Corp., and the Intramural Research Program of the National Cancer Institute (National Institutes of Health, Project \#BC011313). The authors also thank Dr. Jyoti Jaiswal at the George Washington University School of Medicine for the fruitful discussions.

\section{REFERENCES}

(1) Wu, X.; Liu, H. J.; Liu, J. Q.; Haley, K. N.; Treadway, J. A.; Larson, J. P.; Ge, N. F.; Peale, F.; Bruchez, M. P. Immunofluorescent labeling of cancer marker Her2 and other cellular targets with semiconductor quantum dots. Nat. Biotechnol. 2003, 21, 41-46.

(2) Jaiswal, J. K.; Mattoussi, H.; Mauro, J. M.; Simon, S. M. Longterm multiple color imaging of live cells using quantum dot bioconjugates. Nat. Biotechnol. 2003, 21, 47-51.

(3) Dahan, M.; Levi, S.; Luccardini, C.; Rostaing, P.; Riveau, B.; Triller, A. Diffusion dynamics of glycine receptors revealed by singlequantum dot tracking. Science 2003, 302, 442-445.

(4) Alivisatos, P. The use of nanocrystals in biological detection. Nat. Biotechnol. 2004, 22, 47-52.

(5) Medintz, I. L.; Uyeda, H.; Goldman, E.; Mattoussi, H. Quantum dot bioconjugates for imaging, labelling and sensing. Nat. Mater. 2005, 4, 435-446.

(6) Michalet, X.; Pinaud, F.; Bentolila, L.; Tsay, J.; Doose, S.; Li, J.; Sundaresan, G.; Wu, A.; Gambhir, S.; Weiss, S. Quantum dots for live cells, in vivo imaging, and diagnostics. Science 2005, 307, 538-544.

(7) Saha, K.; Agasti, S. S.; Kim, C.; Li, X. N.; Rotello, V. M. Gold Nanoparticles in Chemical and Biological Sensing. Chem. Rev. 2012, 112, 2739-2779.

(8) Mattoussi, H.; Palui, G.; Na, H. B. Luminescent quantum dots as platforms for probing in vitro and in vivo biological processes. $A d v$. Drug Delivery Rev. 2012, 64, 138-166.

(9) Lee, J.; Sharei, A.; Sim, W. Y.; Adamo, A.; Langer, R.; Jensen, K. F.; Bawendi, M. G. Nonendocytic Delivery of Functional Engineered Nanoparticles into the Cytoplasm of Live Cells Using a Novel, HighThroughput Microfluidic Device. Nano Lett. 2012, 12, 6322-6327.

(10) Zhou, J.; Yang, Y.; Zhang, C.-Y. Toward Biocompatible Semiconductor Quantum Dots: From Biosynthesis and Bioconjugation to Biomedical Application. Chem. Rev. 2015, 115, 11669-11717.

(11) Pinaud, F.; Clarke, S.; Sittner, A.; Dahan, M. Probing cellular events, one quantum dot at a time. Nat. Methods 2010, 7, 275-285.

(12) Resch-Genger, U.; Grabolle, M.; Cavaliere-Jaricot, S.; Nitschke, R.; Nann, T. Quantum dots versus organic dyes as fluorescent labels. Nat. Methods 2008, 5, 763-775.

(13) Grabolle, M.; Spieles, M.; Lesnyak, V.; Gaponik, N.; Eychmuller, A.; Resch-Genger, U. Determination of the Fluorescence Quantum Yield of Quantum Dots: Suitable Procedures and Achievable Uncertainties. Anal. Chem. 2009, 81, 6285-6294.

(14) Zrazhevskiy, P.; Sena, M.; Gao, X. H. Designing multifunctional quantum dots for bioimaging, detection, and drug delivery. Chem. Soc. Rev. 2010, 39, 4326-4354.

(15) Dubertret, B.; Skourides, P.; Norris, D. J.; Noireaux, V.; Brivanlou, A. H.; Libchaber, A. In vivo imaging of quantum dots encapsulated in phospholipid micelles. Science 2002, 298, 1759-1762.

(16) Derfus, A. M.; Chan, W. C. W.; Bhatia, S. N. Intracellular delivery of quantum dots for live cell labeling and organelle tracking. Adv. Mater. 2004, 16, 961-966.

(17) Delehanty, J. B.; Mattoussi, H.; Medintz, I. L. Delivering quantum dots into cells: strategies, progress and remaining issues. Anal. Bioanal. Chem. 2009, 393, 1091-1105.

(18) Muroski, M. E.; Carnevale, K. J. F.; Riskowski, R. A.; Strouse, G. F. Plasmid Transfection in Mammalian Cells Spatiotemporally Tracked by a Gold Nanoparticle. ACS Nano 2015, 9, 124-133.

(19) Torchilin, V. P. Tat peptide-mediated intracellular delivery of pharmaceutical nanocarriers. Adv. Drug Delivery Rev. 2008, 60, 548558.

(20) Delehanty, J. B.; Medintz, I. L.; Pons, T.; Brunel, F. M.; Dawson, P. E.; Mattoussi, H. Self-Assembled Quantum Dot-Peptide Bioconjugates for Selective Intracellular Delivery. Bioconjugate Chem. 2006, 17, 920-927.

(21) Delehanty, J. B.; Bradburne, C. E.; Boeneman, K.; Susumu, K.; Farrell, D.; Mei, B. C.; Blanco-Canosa, J. B.; Dawson, G.; Dawson, P. E.; Mattoussi, H.; Medintz, I. L. Delivering quantum dot-peptide bioconjugates to the cellular cytosol: escaping from the endolysosomal system. Integr. Biol. 2010, 2, 265-277. 
(22) Medintz, I. L.; Pons, T.; Delehanty, J. B.; Susumu, K.; Brunel, F. M.; Dawson, P. E.; Mattoussi, H. Intracellular Delivery of Quantum Dot-Protein Cargos Mediated by Cell Penetrating Peptides. Bioconjugate Chem. 2008, 19, 1785-1795.

(23) Ruan, G.; Agrawal, A.; Marcus, A. I.; Nie, S. Imaging and tracking of tat peptide-conjugated quantum dots in living cells: new insights into nanoparticle uptake, intracellular transport, and vesicle shedding. J. Am. Chem. Soc. 2007, 129, 14759-14766.

(24) Chou, L. Y. T.; Ming, K.; Chan, W. C. W. Strategies for the intracellular delivery of nanoparticles. Chem. Soc. Rev. 2011, 40, 233245.

(25) Boeneman, K.; Delehanty, J. B.; Blanco-Canosa, J. B.; Susumu, K.; Stewart, M. H.; Oh, E.; Huston, A. L.; Dawson, G.; Ingale, S.; Walters, R.; Domowicz, M.; Deschamps, J. R.; Algar, W. R.; Dimaggio, S.; Manono, J.; Spillmann, C. M.; Thompson, D.; Jennings, T. L.; Dawson, P. E.; Medintz, I. L. Selecting improved peptidyl motifs for cytosolic delivery of disparate protein and nanoparticle materials. ACS Nano 2013, 7, 3778-3796.

(26) Liu, B. R.; Lo, S.-Y.; Liu, C.-C.; Chyan, C.-L.; Huang, Y.-W.; Aronstam, R. S.; Lee, H.-J. Endocytic Trafficking of Nanoparticles Delivered by Cell-penetrating Peptides Comprised of Nona-arginine and a Penetration Accelerating Sequence. PLoS One 2013, 8, No. e67100.

(27) Derivery, E.; Bartolami, E.; Matile, S.; Gonzalez-Gaitan, M. Efficient Delivery of Quantum Dots into the Cytosol of Cells Using Cell-Penetrating Poly(disulfide)s. J. Am. Chem. Soc. 2017, 139, 10172-10175.

(28) Gasparini, G.; Bang, E.-K.; Montenegro, J.; Matile, S. Cellular uptake: lessons from supramolecular organic chemistry. Chem. Commun. 2015, 51, 10389-10402.

(29) Gaspar, D.; Veiga, A. S.; Sinthuvanich, C.; Schneider, J. P.; Castanho, M. A. R. B. Anticancer Peptide SVS-1: Efficacy Precedes Membrane Neutralization. Biochemistry 2012, 51, 6263-6265.

(30) Medina, S. H.; Schneider, J. P. Cancer cell surface induced peptide folding allows intracellular translocation of drug. J. Controlled Release 2015, 209, 317-326.

(31) Clapp, A. R.; Goldman, E. R.; Mattoussi, H. Capping of CdSe$\mathrm{ZnS}$ quantum dots with DHLA and subsequent conjugation with proteins. Nat. Protoc. 2006, 1, 1258-1266.

(32) Zeng, B.; Palui, G.; Zhang, C.; Zhan, N.; Wang, W.; Ji, X.; Chen, B.; Mattoussi, H. Characterization of the Ligand Capping of Hydrophobic CdSe-ZnS Quantum Dots Using NMR Spectroscopy. Chem. Mater. 2018, 30, 225-238.

(33) Palui, G.; Avellini, T.; Zhan, N.; Pan, F.; Gray, D.; Alabugin, I.; Mattoussi, H. Photoinduced Phase Transfer of Luminescent Quantum Dots to Polar and Aqueous Media. J. Am. Chem. Soc. 2012, 134, 16370-16378.

(34) Wang, W.; Kapur, A.; Ji, X.; Safi, M.; Palui, G.; Palomo, V.; Dawson, P. E.; Mattoussi, H. Photoligation of an Amphiphilic Polymer with Mixed Coordination Provides Compact and Reactive Quantum Dots. J. Am. Chem. Soc. 2015, 137, 5438-5451.

(35) Zhan, N.; Palui, G.; Kapur, A.; Palomo, V.; Dawson, P. E.; Mattoussi, H. Controlling the Architecture, Coordination, and Reactivity of Nanoparticle Coating Utilizing an Amino Acid Central Scaffold. J. Am. Chem. Soc. 2015, 137, 16084-16097.

(36) Sinthuvanich, C.; Veiga, A. S.; Gupta, K.; Gaspar, D.; Blumenthal, R.; Schneider, J. P. Anticancer $\beta$-Hairpin Peptides: Membrane-Induced Folding Triggers Activity. J. Am. Chem. Soc. 2012, 134, 6210-6217.

(37) Zhang, C.; Palui, G.; Zeng, B.; Zhan, N.; Chen, B.; Mattoussi, $\mathrm{H}$. Non-Invasive Characterization of the Organic Coating of Biocompatible Quantum Dots Using Nuclear Magnetic Resonance Spectroscopy. Chem. Mater. 2018, 30, 3454-3466.

(38) Mattoussi, H.; Cumming, A. W.; Murray, C. B.; Bawendi, M. G.; Ober, R. Properties of CdSe nanocrystal dispersions in the dilute regime: Structure and interparticle interactions. Phys. Rev. B 1998, 58, $7850-7863$.
(39) Anas, A.; Okuda, T.; Kawashima, N.; Nakayama, K.; Itoh, T.; Ishikawa, M.; Biju, V. Clathrin-mediated endocytosis of quantum dotpeptide conjugates in living cells. ACS Nano 2009, 3, 2419-2429.

(40) Heuser, J. E.; Anderson, R. G. Hypertonic media inhibit receptor-mediated endocytosis by blocking clathrin-coated pit formation. J. Cell Biol. 1989, 108, 389-400.

(41) Daukas, G.; Zigmond, S. H. Inhibition of receptor-mediated but not fluid-phase endocytosis in polymorphonuclear leukocytes. J. Cell Biol. 1985, 101, 1673-1679.

(42) Kapur, A.; Medina, S. H.; Wang, W.; Palui, G.; Schneider, J. P.; Mattoussi, H. Intracellular Delivery of Gold Nanocolloids Promoted by a Chemically Conjugated Anticancer Peptide. ACS Omega 2018, 3, 12754-12762.

(43) Verma, A.; Uzun, O.; Hu, Y.; Hu, Y.; Han, H.-S.; Watson, N.; Chen, S.; Irvine, D. J.; Stellacci, F. Surface-structure-regulated cellmembrane penetration by monolayer-protected nanoparticles. Nat. Mater. 2008, 7, 588.

(44) Leatherdale, C. A.; Woo, W. K.; Mikulec, F. V.; Bawendi, M. G. On the absorption cross section of CdSe nanocrystal quantum dots. J. Phys. Chem. B 2002, 106, 7619-7622.

(45) Dabbousi, B. O.; RodriguezViejo, J.; Mikulec, F. V.; Heine, J. R.; Mattoussi, H.; Ober, R.; Jensen, K. F.; Bawendi, M. G. (CdSe)ZnS core-shell quantum dots: Synthesis and characterization of a size series of highly luminescent nanocrystallites. J. Phys. Chem. B 1997, 101, 9463-9475.

(46) Hermanson, G. T. Bioconjugate Techniques, 3rd ed.; Academic press, 2013; pp 1-1146.

(47) Jaiswal, J. K.; Mattoussi, H.; Mauro, J. M.; Simon, S. M. Longterm multiple color imaging of live cells using quantum dot bioconjugates. Nat. Biotechnol. 2003, 21, 47-51.

(48) Drin, G.; Cottin, S.; Blanc, E.; Rees, A. R.; Temsamani, J. Studies on the Internalization Mechanism of Cationic Cellpenetrating Peptides. J. Biol. Chem. 2003, 278, 31192-31201.

(49) Massodi, I.; Bidwell, G. L.; Raucher, D. Evaluation of cell penetrating peptides fused to elastin-like polypeptide for drug delivery. J. Controlled Release 2005, 108, 396-408. 